\title{
Effets des techniques de coupe sur la croissance et le nombre des rejets dans un taillis de châtaignier (Castanea sativa Mill.)
}

\author{
A Cabanettes*, L Pagès** \\ Avec la collaboration technique de A Moreau et JP Château
}

INRA, station de sylviculture Ardon, 45160 Olivet, France

(Reçu le 26 septembre 1988; accepté le 1er février 1989)

\begin{abstract}
Résumé - Cette étude a pour objectif de comparer l'effectif et la croissance, en diamètre et en longueur, de rejets de châtaignier au terme de leur première année de végétation, selon différentes modalités de coupe du peuplement initial. Ces modalités concernent 2 outils de coupe, la tronçonneuse et la hache, et, pour ce dernier, 2 hauteurs de coupe différentes $(10$ et $30 \mathrm{~cm})$. Les résultats obtenus montrent qu'en ce qui concerne l'outil hache, la coupe haute tend à entraîner l'apparition de rejets de plus petit diamètre et, corrélativement, de plus petite longueur que la coupe basse. Pour une même hauteur de coupe, l'utilisation de la hache entraîne la croissance de rejets significativement plus longs (mais pas plus gros) que ceux obtenus après coupe à la tronçonneuse. L'effet spécifique de l'outil de coupe sur la seule longueur se retrouve au niveau de la relation statistique liant longueur et diamètre des rejets. Enfin, l'effectif des rejets âgés d'un an est surtout significativement diminué par l'utilisation de la tronçonneuse, par rapport au résultat obtenu à l'aide de la hache. On formule des hypothèses sur la nature et sur l'origine physiologique des phénomènes observés
\end{abstract}

croissance / taillis / Castanea sativa / recépage / hache / tronçonneuse

Summary - Effects of cutting techniques on growth and number of sprouts in a chestnut (Castanea sativa Mill.) coppice. This study was aimed at comparing the effect of the method of cutting the initial coppice on the growth (diameter and length) and number of one year old sprouts. The cutting methods were: 2 cutting tools, axe and chainsaw and 2 heights $(10$ and $30 \mathrm{~cm})$ for the axe treatment (fig. 1). The results obtained showed that for axe treatment at $30 \mathrm{~cm}$, the sprouts are smaller and thinner than for that at $10 \mathrm{~cm}$. For low cutting, chestnut sprouts are significantly longer (but no larger) with an axe than with a chainsaw (table II). This effect is also visible on the statistical relationship between length and diameter of the sprouts (table IV). Finally, the number of one year old sprouts is chiefly lower in the chainsaw treatment (fig. 4). Biological and mechanical hypotheses are formulated to explain these results.

growth / coppice / Castanea sativa / coppicing / axe / chainsaw

* Adresse actuelle: INRA, Station de recherches forestières, domaine de l'hermitage, Pierroton, 33610 Cestas, France.

** Adresse actuelle: INRA, Station d'agronomie, domaine Saint-Paul, BP 91, 84140 Montfavet, France. 


\section{INTRODUCTION}

Le régime forestier du taillis est caractérisé par la fréquence relativement élevée des coupes successives qui ont lieu sur le même peuplement: les recépages sont effectués tous les 10 à 30 ans en moyenne, et concernent la totalité des individus dans le cas du taillis simple. De ce fait, les modalités d'exploitation actuelles et passées (durée des rotations ou âge ou dimension d'exploitation, saison, outil et hauteur de coupe, mode de récolte) revêtent une importance particulière dans ce mode de sylviculture. Par leur influence positive ou négative sur l'évolution spatiale et temporelle de l'appareil racinaire permanent, des bourgeons dormants aériens, des liaisons tiges/racines et du sol, ces modalités contrôlent la densité d'ensouchement, l'âge physiologique et l'efficacité de fonctionnement des souches, et donc la production ligneuse aérienne pour une espèce et un milieu naturel initial donnés. La synthèse de Blake et Raitanen (1981), les résultats obtenus par Solomon et Blum (1967), Johnson (1975), ainsi que des travaux plus récents (Riedacker et al, 1985; Harrington, 1984) en témoignent largement. De plus, les bases physiologiques de ces effets commencent à être mises en lumière (Blake, 1981; Taylor et al, 1982; Rinne et al, 1987).

Aussi, dans un objectif de modélisation de la croissance des taillis, la prise en compte des modalités d'exploitation actuelles et passées comme variables d'entrée du modèle est une nécessité qui apparaît lorsque l'on désire établir un modèle au niveau peuplement basé sur un échantillon géographiquement très variable. Les variables explicatives classiques de la production utilisées en futaie (hauteur dominante, classe de production, âge, et même densité de peuplement) se révèlent alors insuffisantes, laissant subsister une importante variabilité non expli- quée par le modèle (Bédéneau, 1988). Ce phénomène n'apparaît plus lorsque l'on s'adresse à une même région à pratiques sylvicoles homogènes sur une espèce donnée (Pagès, 1986).

Mais les modalités d'exploitation du taillis pratiquées en France varient à la fois spatialement et temporellement d'une manière très difficile à contrôler scientifiquement. Aussi est-il utile d'expérimenter analytiquement, sur des périodes de temps suffisantes, pour pouvoir en déceler et en mesurer les effets spécifiques durables sur la croissance et la production des taillis, et en déduire d'éventuelles conclusions pratiques.

Parmi les modalités d'exploitation, les effets de la hauteur de coupe, et surtout de la nature de l'outil de coupe sont beaucoup moins étudiés que ceux de la durée de rotation, de la dimension et de l'âge d'exploitation et de la saison de coupe sur la repousse du taillis. De plus, les quelques références existantes font apparaître des résultats peu nets ou contradictoires (Parade, 1860; Blake et Raitanen, 1981; Phillips, 1970; Crist et al, 1983; Harrington, 1984; Khan et Tripathi, 1986; El Houri Ahmed, 1977; Bélanger, 1979; Debell, 1972).

Aussi, l'étude présentée ici a-t-elle pour objectif la mise en évidence d'éventuels effets séparés sur la croissance et sur le nombre de rejets de 2 modalités de coupe associées dans la gestion forestière:

- le type d'outil: la hache et la tronçonneuse (ou scie à chaîne), qui diffèrent à la fois par l'effet direct sur le bois (fente ou broyage) et des effets indirects (chauffage, angle de la surface de coupe par rapport à l'horizontale, ébranlement de la souche);

- la hauteur de coupe: $10 \mathrm{~cm}$ et $30 \mathrm{~cm}$ audessus du sol; ces 2 niveaux sont situés dans la gamme des variations pratiques courantes. Pour la tronçonneuse, dont la hauteur de coupe est plus maîtrisable, on n'a expérimenté que la hauteur de $10 \mathrm{~cm}$. 
Après l'analyse qui a concerné la variable «hauteur du maître-rejet des cépées" (Cabanettes et Pagès, 1986), nous présentons ici les résultats obtenus pour la croissance en diamètre et en longueur de l'ensemble des rejets.

\section{MATÉRIEL ET MÉTHODES}

Les caractéristiques du dispositif ont été présentées en détail dans un précédent article (Cabanettes et Pagès, 1986). Rappelons seulement ici qu'il est situé au cœur de la châtaigneraie limousine, dans le secteur des efeuillardiers" où le taillis de châtaignier est exploité à rotations de 6-10 ans. Le peuplement initial était un taillis de châtaigniers rigoureusement pur et équienne âgé de 11 ans. L'objet d'étude est ici la repousse âgée d'un an issue d'une coupe à blanc effectuée en décembre 1983. Les 3 traitements expérimentés sont: la coupe à la hache à $30 \mathrm{~cm}$, la coupe à la hache à 10 $\mathrm{cm}$, la coupe à la tronçonneuse à $10 \mathrm{~cm}$. Le dispositif est organisé selon 3 blocs (ou répétitions); dans chaque bloc, chaque traitement est pratiqué sur 2 placeaux de $200 \mathrm{~m}^{2}$ environ.

Avant la coupe initiale de décembre 1983 ont été déterminés le nombre de tiges vivantes et le diamètre moyen au sol pour chaque cépée du dispositif. Les mesures de la repousse d'un an ont porté sur la totalité des rejets de 24 cépées échantillons. Le choix de ces cépées a été fait de façon aléatoire sous contraintes, les contraintes étant d'obtenir 8 cépées par traitement, également réparties dans les 2 classes de nombre initial de tiges par cépée situées de part et d'autre de la moyenne (soit 15 tiges par cépée), et d'avoir un minimum de 2 cépées par bloc (et par traitement). La contrainte de l'égale répartition des cépées selon le nombre initial de tiges à l'intérieur de chaque traitement a été

Tableau I. Répartition dans le dispositif et caractéristiques des cépées échantillons.

\begin{tabular}{|c|c|c|c|c|c|c|c|}
\hline $\begin{array}{l}N^{\circ} d u \\
\text { bloc }\end{array}$ & traitement & $\begin{array}{c}N^{\circ} d e \\
\text { placeau }\end{array}$ & $\begin{array}{l}N^{\circ} \text { de } \\
\text { cépée }\end{array}$ & $\begin{array}{l}\text { Nombre } \\
\text { d'étocs }\end{array}$ & $\begin{array}{c}\text { Encom- } \\
\text { brement } \\
\text { moyen (DM) }\end{array}$ & $\begin{array}{c}\text { Nombre } \\
\text { de rejets } \\
1 \text { an }\end{array}$ & $\begin{array}{l}\text { Hauteur } \\
\text { du maître } \\
\text { rejet (CM) }\end{array}$ \\
\hline \multirow[t]{10}{*}{1} & Hache bas & 02 & 406 & 10 & 10 & 40 & 89 \\
\hline & & 02 & 532 & 35 & 25 & 205 & 138 \\
\hline & & 04 & 480 & 51 & 22 & 252 & 155 \\
\hline & Hache haut & 01 & 248 & 9 & 8 & 64 & 130 \\
\hline & & 03 & 331 & 1 & 8 & 25 & 122 \\
\hline & & 03 & 430 & 17 & 20 & 150 & 124 \\
\hline & Tronçonneuse & 05 & 766 & 30 & 18 & 122 & 124 \\
\hline & & 05 & 790 & 2 & 5 & 15 & 71 \\
\hline & & 06 & 774 & 7 & 9 & 41 & 125 \\
\hline & & 06 & 792 & 16 & 14 & 78 & 126 \\
\hline \multirow[t]{6}{*}{2} & Hache bas & 09 & 327 & 22 & 18 & 120 & 205 \\
\hline & & 12 & 719 & 11 & 10 & 54 & 181 \\
\hline & Hache haut & 10 & 517 & 10 & 7 & 47 & 113 \\
\hline & & 11 & 692 & 36 & 18 & 188 & 146 \\
\hline & Tronçonneuse & 07 & 77 & 6 & 12 & 39 & 116 \\
\hline & & 08 & 319 & 3 & 4 & 22 & 130 \\
\hline \multirow[t]{8}{*}{3} & Hache bas & 17 & 569 & 6 & 10 & 41 & 184 \\
\hline & & 17 & 587 & 19 & 19 & 149 & 240 \\
\hline & & 18 & 608 & 12 & 16 & 103 & 208 \\
\hline & Hache haut & 13 & 7 & 28 & 32 & 201 & 223 \\
\hline & & 13 & 20 & 24 & 30 & 267 & 220 \\
\hline & & 14 & 285 & 14 & 14 & 59 & 157 \\
\hline & Tronçonneuse & 15 & 395 & 21 & 25 & 117 & 181 \\
\hline & & 16 & 558 & 38 & 20 & 126 & 173 \\
\hline
\end{tabular}


respectée afin de mieux maîtriser un éventuel effet de cette variable sur la croissance des rejets, susceptible de se superposer ou d'interagir avec les effets des modalités de coupe pratiquées. Le grand nombre de rejets à mesurer par cépées (tableau I) n'a toutefois pas permis de prendre en compte un nombre de cépées suffisant pour que le nombre initial de tiges puisse être inclus de manière efficace dans les modèles d'analyse des données. La répartition et les caractéristiques de l'échantillon sont indiquées dans le tableau I. Sur chaque rejet vivant ont été mesurés le diamètre basal (au-dessus de l'empattement) au dixième de millimètre et la longueur totale à partir de la naissance du rejet sur la souche, au centimètre.

L'analyse des données a consisté d'une part en une analyse de variance des 2 variables diamètre et longueur selon les niveaux des facteurs bloc et traitement de coupe, et d'autre part en une comparaison selon ces mèmes facteurs des liaisons statistiques existant entre les 2 variables. Les calculs d'analyse de variance ont pu être effectués en prenant en compte le caractère "déséquilibré et non-orthogonal" (non-proportionnalité des effectifs de rejets d'un bloc à l'autre ou d'un traitement à l'autre) du plan d'expérience grâce au programme ANVARM du logiciel AMANCE (Bachacou et al, 1981). La construction des modèles statistiques reliant le diamètre et la longueur des rejets a été réalisée à l'aide du logiciel GENSTAT (Astier et al, 1982). Après vérification a posteriori du caractère gaussien des résidus, la comparaison de ces modèles a été effectuée en confrontant les sommes de carrés d'écarts résiduels des modèles à l'aide d'un test $F$, selon la méthode décrite par Snedecor et Cochran (1957).

\section{RÉSULTATS}

\section{Croissance des rejets}

\section{Analyse séparée des variables dia- mètre et longueur}

Les résultats moyens concernant les mesures de diamètre et de longueur des rejets sont indiqués avec leur dispersion, par bloc et par traitement, dans la figure 1. Ces résultats suggérant un certain nombre de tendances, on a effectué sur l'ensemble des données brutes une analyse de variance multivariable répondant à

\footnotetext{
* Les distributions des variables étant intermédiaires entre celles des lois normale et lognormale, cette même analyse a été tentée également sur les valeurs logarithmiques des données. Les résultats ont été identiques.
}

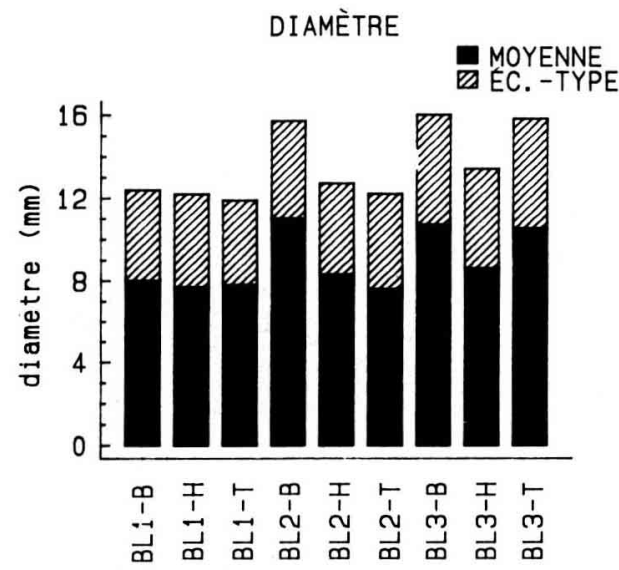

LONGUEUR

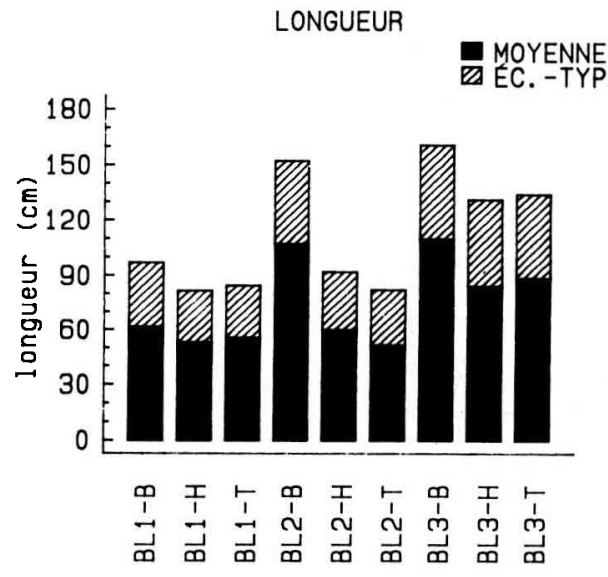

Fig. 1. Valeurs moyennes et dispersions du diamètre et de la longueur des rejets pour chacune des unités expérimentales. $\mathrm{BL}=$ bloc, suivi de son numéro; $\mathrm{B}=$ coupe hache bas; $\mathrm{H}=$ coupe hache haut; $\mathrm{T}=$ coupe tronçonneuse. Le nombre moyen de rejets par unité expérimentale est de 253 rejets. 
Tableau II. Résultats de l'analyse de variance séparée des deux variables diamètre et longueur. $F=$ Valeur calculée de la variable $F$ de Fisher; ${ }^{\star *}=$ valeur significative au risque de $\uparrow \%$. Les effets indiquées pour chaque bloc, traitement et interaction bloc/traitement correspondent respectivement aux valeurs ajustées $A, B$ et $A B$ du modèle analysé, soit: $Y=M U+A+B+A B$ où $M U$ est la moyenne générale de la variable $Y$ (diamètre ou longueur) pour le dispositif. Les effets sont exprimés en $\mathrm{mm}$ pour le diamètre et en $\mathrm{cm}$ pour la longueur. Pour chaque variable et chaque effet (ou chaque traitement pour les interactions), seuls les chiffres n'ayant pas de lettres communes en exposant diffèrent significativement entre eux au seuil de $5 \%$ ou $1 \%$.

\begin{tabular}{lrr}
\hline & Diamètre & Longueur \\
\hline Effet bloc & $F=52,31^{\star \star}$ & $F=233,0^{\star \star}$ \\
Bloc 1 & $-1,22^{\mathrm{a}}$ & $-20,91^{\mathrm{a}}$ \\
Bloc 2 & $+0,49^{\mathrm{b}}$ & $+1,21^{\mathrm{b}}$ \\
Bloc 3 & $+0,92^{\mathrm{b}}$ & $+18,96^{\mathrm{c}}$ \\
Effet traitement & $F=26,8^{\star \star}$ & $F=82,7^{\star \star}$ \\
Hache bas & $+0,76^{\mathrm{a}}$ & $+13,40^{\mathrm{a}}$ \\
Hache haut & $-0,83^{\mathrm{b}}$ & $-9,99^{\mathrm{b}}$ \\
Tronçonneuse & $+0,17^{\mathrm{ab}}$ & $-5,10^{\mathrm{b}}$ \\
Interaction & $F=8,88^{\star \star}$ & $F=20,4^{\star \star}$ \\
Hache bas $\times$ Bloc 1 & $-0,40^{\mathrm{a}}$ & $-6,83^{\mathrm{a}}$ \\
Hache bas $\times$ Bloc 2 & $+0,94^{\mathrm{a}}$ & $+16,72^{\mathrm{b}}$ \\
Hache bas $\times$ Bloc 3 & $+0,13^{\mathrm{a}}$ & $+1,61^{\mathrm{a}}$ \\
Hache haut $\times$ Bloc 1 & $+0,86^{\mathrm{a}}$ & $+8,01^{\mathrm{a}}$ \\
Hache haut Bloc 2 & $-0,17^{\mathrm{a}}$ & $-7,08^{\mathrm{a}}$ \\
Hache haut $\times$ Bloc 3 & $-0,31^{\mathrm{a}}$ & $-0,48^{\mathrm{a}}$ \\
Tronçonneuse $\times$ Bloc 1 & $-0,02^{\mathrm{ab}}$ & $+5,72^{\mathrm{a}}$ \\
Tronçonneuse $\times$ Bloc 2 & $-2,01^{\mathrm{b}}$ & $-20,42^{\mathrm{b}}$ \\
Tronçonneuse $\times$ Bloc 3 & $+0,53^{\mathrm{a}}$ & $-0,90^{\mathrm{a}}$ \\
\hline
\end{tabular}

un modèle classique à deux facteurs croisés et à effets fixés*. Dans le tableau II sont indiqués les résultats globaux de l'analyse de variance (test $F$ ), ainsi que les valeurs moyennes ajustées des effets bloc et traitement et de l'interaction pour chaque couple traitement/bloc. Les moyennes ont été comparées 2 à 2 à l'aide du test du $t$-corrigé dit de Bonferroni (Bachacou et al, 1981; Dagnélie, 1970).

On constate que les effets traitement et bloc sont significatifs, le premier étant moins important que le second, et tous 2 étant plus nets sur la longueur que sur le diamètre; il existe une interaction significative, surtout pour la longueur, qui signifie notamment qu'il n'est pas toujours pos- sible de définir un effet traitement donné moyen pour l'ensemble des blocs.

La comparaison individuelle des effets blocs révèle qu'ils se distinguent davantage pour la longueur que pour le diamètre, avec dans ce dernier cas similitude des blocs 2 et 3 ; le classement est: bloc $3>$ bloc $2>$ bloc 1 .

La comparaison individuelle des traitements fait apparaître, pour le diamètre, un effet de la hauteur de coupe pour l'instrument hache (coupe basse > coupe haute) et une absence d'influence de l'outil de coupe, et, pour la longueur, une influence conjointe de la hauteur de coupe (allant dans le même sens que pour le diamètre) et de l'instrument de coupe (effet positif de 
la hache et effet plutôt dépressif de la tronçonneuse), le premier effet étant le plus important.

Si l'on analyse l'interaction globale, on voit qu'elle concerne surtout le bloc 2 , où se rencontrent les interactions les plus élevées pour les modalités tronçonneuse (diamètre et surtout longueur) et hache bas (longueur): les effets y sont principalement sous-évalués par le modèle, qu'ils soient globalement positifs (hache bas pour la longueur) ou globalement négatifs (tronçonneuse pour la longueur). Les interactions vont dans le même sens que les effets principaux.

\section{Analyse de la relation entre longueur et diamètre}

Au cours de l'étude séparée des 2 variables, nous avons pu observer que la longueur était généralement l'objet d'effets plus importants de la part des facteurs que ne l'était le diamètre. Du fait de la forte corrélation globale existant entre ces deux variables (Fig. 2) et des tendances observables sur la figure 3 , nous avons recherché s'il existait des effets spécifiques des facteurs étudiés sur la seule longueur (L) des rejets, conditionnelle-

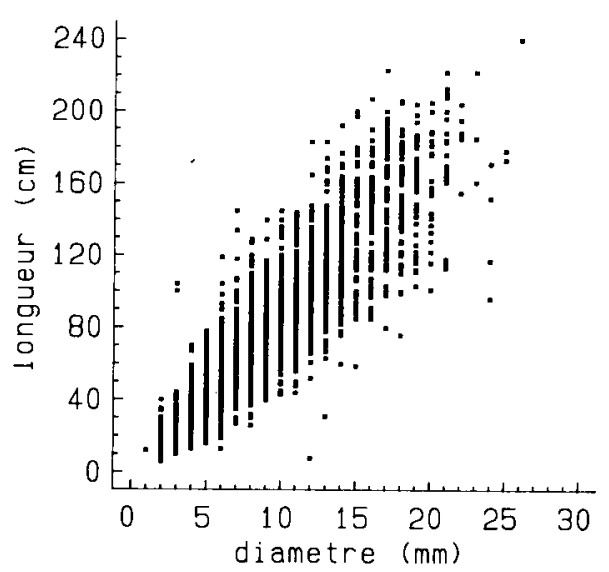

Fig. 2. Représentation par points des couples (Iongueur, diamètre) pour l'ensemble des 2522 rejets. Le coefficient de corrélation linéaire de Bravais-Pearson a pour valeur 0,898 .

ment à leur diamètre (D). La figure 2 suggérant une relation de type linéaire entre les 2 variables, nous avons effectué une analyse du modèle de régression:

$$
L=a D+b
$$

selon les facteurs étudiés. Pour ce faire, le modèle général (1) et les 5 modèles suivants ont été construits puis comparés, la
A - INFLUENCE DU BLOC

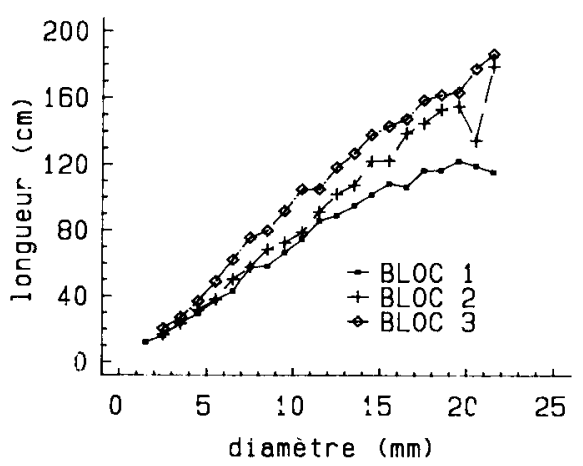

B - influence du tRaitement

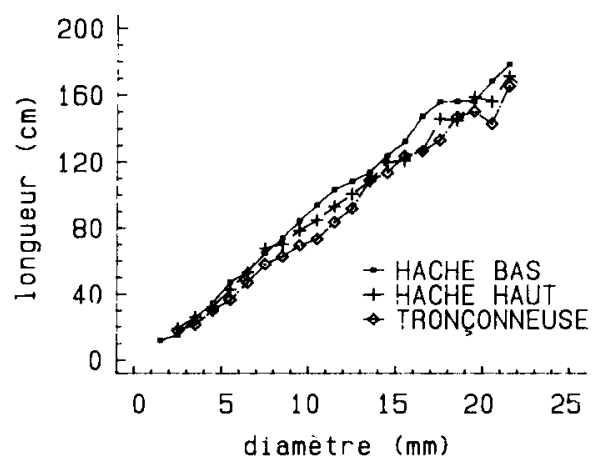

Fig. 3. Représentation selon le bloc (A) ou le traitement (B) des liaisons entre la longueur et le diamètre des 2522 rejets appartenant aux 24 cépées-échantillons. Chaque point représente la moyenne d'environ 50 observations. 
Tableau III. Résultats des comparaisons des 6 modèles de régression de la longueur sur le diamètre. $\left(^{\star \star}\right)$ valeur de $F$ significative au seuil de $1 \%$; (NS) valeur de $F$ non significative au seuil de $5 \%$.

\begin{tabular}{|c|c|c|c|c|}
\hline $\begin{array}{l}\text { Hypothèse } \\
\text { testée }\end{array}$ & $\begin{array}{l}\text { Modèles } \\
\text { comparés }\end{array}$ & $\begin{array}{l}\text { Nombre de } \\
\text { degrés de } \\
\text { liberté }\end{array}$ & F calculé & Conclusions \\
\hline $\begin{array}{l}\text { L'ordonnée à l'origine } \\
\text { est nulle }\end{array}$ & (1) ET (2) & 1 et 2519 & 0,245 (NS) & ordonnée à l'origine nulle \\
\hline $\begin{array}{l}\text { La pente ne dépend pas } \\
\text { du bloc }\end{array}$ & (2) ET (3) & 2 et 2518 & $375\left(^{* \star}\right)$ & la pente dépend du bloc \\
\hline $\begin{array}{l}\text { La pente ne dépend pas } \\
\text { du traitement }\end{array}$ & (2) ET (4) & 2 et 2518 & $46,0\left(^{\star \star}\right)$ & la pente dépend du traitement \\
\hline $\begin{array}{l}\text { La prise en compte des } \\
\text { effets bloc et traitement } \\
\text { indépendants est meilleure } \\
\text { que celle du seul effet bloc }\end{array}$ & (3) ET (5) & 2 et 2516 & $121,4\left(^{\star \star}\right)$ & $\begin{array}{l}\text { les deux effets bloc + traitement } \\
\text { sont significatifs }\end{array}$ \\
\hline $\begin{array}{l}\text { L'interaction entre bloc et } \\
\text { traitement n'est pas à } \\
\text { prendre en compte }\end{array}$ & (5) ET (6) & 4 et 2512 & $89,9(* *)$ & $\begin{array}{l}\text { interaction bloc } \times \text { traitement } \\
\text { significative }\end{array}$ \\
\hline
\end{tabular}

variance résiduelle étant stabilisée par une pondération en $1 / \sqrt{ } \mathrm{L}$ :

(2) $L=a^{\star} D$ où l'ordonnée à l'origine $b$ est nulle;

(3) $L=a_{b l o c} \cdot D$ où la pente dépend du bloc;

(4) $L=a_{\text {trait }} \cdot D$ où la pente dépend du traitement;

(5) $L=\left(a_{\text {bloc }}+a_{\text {trait }}\right) \cdot D$ où les effets bloc et traitement sont pris en compte indépendamment;

(6) $L=a_{\text {bloc,trait }}$ D où l'interaction bloc/traitement est prise en compte.

Les résultats sont indiqués dans le tableau III. On voit que la pente de la régression linéaire liant la longueur au diamètre dépend à la fois du bloc et du traitement de coupe, le premier effet étant nettement le plus important. Toutefois, il y a interaction entre ces deux effets, ce qui signifie notamment qu'il n'est pas toujours possible, pour un traitement donné, de définir une pente moyenne indépendante du bloc. Pour mieux cerner la nature et l'ordre de grandeur des effets et de l'interaction, nous avons reporté dans le tableau
IV les diverses pentes fournies par les modèles (3), (4) et (6), calculées par le logiciel GENSTAT, et les avons comparées 2 à 2 à l'aide du test $t$ de Student corrigé. On constate:

- que les effets bloc et traitement diffèrent tous globalement 2 à 2 , avec des pentes classées dans l'ordre:

$$
\text { bloc } 3>\text { bloc } 2>\text { bloc } 1
$$

hache bas $>$ hache haut $>$ tronçonneuse;

- au niveau de l'interaction, que le résultat global ne se retrouve pas toujours: 1) pour les blocs 1 et 2, les traitements hache haut et tronçonneuse ne diffèrent pas significativement, 2) pour le bloc 3 , les traitements hache bas et hache haut ne se distinguent pas significativement;

- que les résultats constant, retrouvé dans les 3 blocs, est que la pente du traitement hache bas est toujours significativement supérieure à la pente du traitement tronçonneuse, ce qui montre un effet significatif de l'instrument de coupe sur la longueur, conditionnellement au diamètre. 
Tableau IV. Comparaison des valeurs des pentes correspondant aux modèles de régression (3) à (6). Pour chaque effet (ou chaque bloc pour les interactions), seuls les chiffres n'ayant pas de lettres communes en exposant diffèrent significativement entre eux au seuil de $5 \%$ ou $1 \%$.

\begin{tabular}{llcl}
\hline & & Pente $(\mathrm{cm} / \mathrm{mm})$ & Écart type de la pente $(\mathrm{cm} / \mathrm{mm})$ \\
\hline Effet bloc & & $7,06^{\mathrm{a}}$ & \\
Bloc 1 & $8,05^{\mathrm{b}}$ & 0,0613 \\
Bloc 2 & $9,30^{\mathrm{c}}$ & 0,1025 \\
Bloc 3 & & 0,0822 \\
Effet traitement & & \\
Hache bas & & $8,66^{\mathrm{a}}$ & 0,0645 \\
Hache haut & & $8,9^{\mathrm{b}}$ & 0,0935 \\
Tronçonneuse & $7,65^{\mathrm{c}}$ & 0,1066 \\
Interaction & & & \\
Bloc 1 & Hache bas & $7,33^{\mathrm{a}}$ & 0,0797 \\
& Hache haut & $6,62^{\mathrm{b}}$ & 0,1402 \\
& Tronçonneuse & $6,92^{\mathrm{b}}$ & 0,1377 \\
Bloc 2 & Hache bas & $9,49^{\mathrm{a}}$ & 0,1401 \\
& Hache haut & $7,03^{\mathrm{b}}$ & 0,2131 \\
& Tronçonneuse & $6,68^{\mathrm{b}}$ & 0,2930 \\
Bloc 3 & Hache bas & $9,88^{\mathrm{a}}$ & 0,1211 \\
& Hache haut & $9,48^{\mathrm{a}}$ & 0,1844 \\
& Tronçonneuse & $8,37^{\mathrm{b}}$ & 0,1916 \\
\hline
\end{tabular}

\section{Nombre de rejets par étoc}

La mise en évidence d'éventuels effets des modalités de coupe sur le nombre de rejets est facilitée si l'on parvient à expliquer une partie de la variabilité de cette grandeur en utilisant les relations qui la lient à d'autres facteurs variant sur le dispositif d'étude. Au niveau de la cépée, le nombre d'étocs est le plus évident de ces facteurs, mais il n'est pas le seul, le nombre de jeunes rejets de châtaigniers portés par un étoc augmentant avec le diamètre de celui-ci (Cabanettes, 1986). Or, on observe que, pour un nombre d'étocs donné, l'écartement des étocs tend à augmenter avec leur diamètre moyen. Le tableau I montre ainsi une tendance moyenne du nombre de rejets par cépée (NR) à s'accroître avec l'encombrement (ENC) de la cépée, pour un nombre d'étocs (NET) donné. Aussi avons-nous utilisé les 2 variables NET et ENC afin d'obtenir une estimation moyenne de NR indépendante des modalités de la dernière coupe. Le modèle suivant, non linéaire pondéré, s'est révélé le plus satisfaisant:

$\mathrm{NR}=1,34 \cdot\left[\mathrm{NET} 0,5 \cdot \mathrm{ENC}^{1,0}\right]+11,0$

avec: nombre d'individus $=24$ et coefficient de corrélation $=0,96$.

Ce modèle moyen a été appliqué aux mêmes cépées échantillons, fournissant pour chacune d'elles un effectif moyen théorique de rejets, qui est confronté à l'effectif observé *: ces comparaisons, qui équivalent à analyser les résidus du modèle (7), sont récapitulées par bloc et

\footnotetext{
* Du fait du trop petit nombre de cépées par bloc et par traitement, et du caractère non licite des analyses de régressions calculées à partir de variables «effectif", il n'a pas été possible ici d'effectuer une comparaison efficace de régressions comme au paragraphe "Analyse de la relation entre longueur et diamètre".
} 


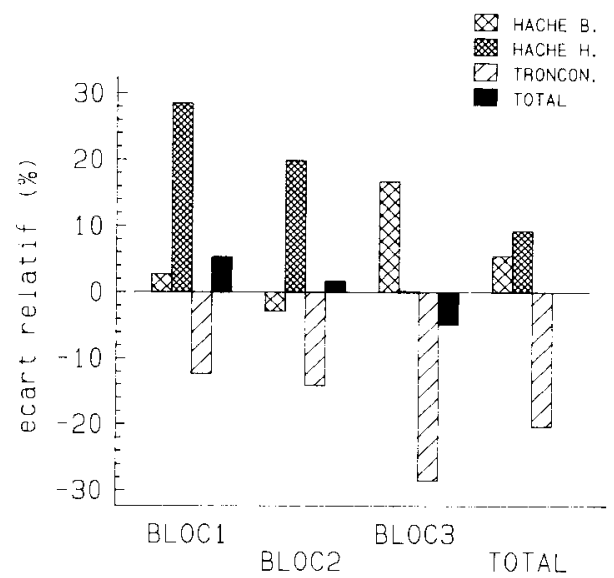

Fig. 4. Distribution par bloc et par traitement des écarts relatifs (NO-NC)/NO entre les effectifs de rejets observés (NO) et les effectifs de rejets calculés à l'aide de l'équation de régression [7] au niveau de chacune des 24 cépées-échantillons (NC).

par traitement sur la figure 4. Les phénomènes les plus marquants se situent au niveau des moyennes marginales.

- pour les modalités de coupe, la comparaison des effectifs observés aux valeurs calculées montre que les effets traitement sur le nombre de rejets se classent dans l'ordre: hache haut > hache bas > tronçonneuse, où seule la modalité tronçonneuse a un effet négatif (effectif observé inférieur à l'effectif théorique);

- pour les blocs, les différences entre effectifs observés et calculés sont relativement faibles; le classement par ordre d'effet positif décroissant est le suivant: bloc $1>$ bloc $2>$ bloc 3 . Le bloc 3 est le seul bloc où l'effectif observé est inférieur à l'effectif théorique.

\section{DISCUSSION - CONCLUSION}

Rappelons tout d'abord les principaux résultats qui ont été obtenus lors de cette étude concernant la repousse âgée d'un an d'un taillis de châtaignier;
- les 3 modalités de coupe expérimentées ont eu un effet significatif sur la croissance en diamètre et en longueur des rejets. Les interactions observées entre blocs et traitements ne remettent pas en cause les résultats globaux, mais l'effet de chaque traitement est modulé selon le bloc, le caractère significatif et le sens des différences observées n'étant pas modifiés. Pour le diamètre, seules les deux modalités de hauteur de coupe de l'outil hache se distinguent significativement l'une de l'autre, entraînant une réduction moyenne (coupe haute) ou une augmentation moyenne (coupe basse) de $0,8 \mathrm{~mm}$ (soit \pm $9 \%$ par rapport au diamètre de l'ensemble des rejets. Pour la longueur, on a d'une part un effet de la hauteur de coupe de même sens que pour le diamètre $(+13,4 \mathrm{~cm}$, soit $+18 \%$ pour la coupe basse; $-10 \mathrm{~cm}$, soit $-13 \%$ pour la coupe haute) et d'autre part un effet, plus faible, de l'outil de coupe $(-5,1 \mathrm{~cm}$, soit $-7 \%$ pour la tronçonneuse);

- en ce qui concerne l'effet des modalités sur le rapport longueur/diamètre, en tenant compte de l'interaction bloc*traitement, on n'observe qu'un effet de l'outil de coupe: pour un diamètre donné, l'outil hache entraîne une augmentation significative moyenne de longueur de $5 \%$ alors que l'utilisation de la tronçonneuse aboutit à une réduction de $7 \%$;

- pour le nombre de rejets, c'est principalement la coupe haute qui entraîne leur augmentation (+9\% par rapport au nombre moyen); à hauteur de coupe égale, la modalité tronçonneuse entraîne une diminution de $-20 \%$, contre une différence de seulement $+5 \%$ pour la modalité hache bas.

On remarquera dans l'ensemble, pour la croissance des rejets, la prédominance des effets bloc sur les effets traitement. II s'agit là d'un phénomène courant dans les expérimentations effectuées en milieu naturel, qui s'est trouvé accentué ici par le fait que le dispositif est situé sur une 
pente, avec étagement des blocs 1 (haut de pente), 2 (mi-pente) et 3 (bas de pente). On peut assimiler dans notre cas l'effet bloc à un effet "fertilité", car il correspond toujours à un classement des effets sur la longueur et sur le diamètre dans l'ordre décroissant: bloc3 > bloc2 > bloc1, les différences entre ces effets étant presque toujours significatives. Au contraire, la variable nombre de rejets est beaucoup moins dépendante du bloc que de la modalité de coupe. D'autre part, pour les variables diamètre et longueur, l'effet hauteur de coupe tend à primer sur celui de l'outil, alors que pour les variables longueur/diamètre et nombre de rejets c'est plutôt l'inverse.

La sensibilité de la croissance en diamètre des rejets à la seule hauteur de coupe révèle sans doute la prédominance des effets de concurrence entre rejets d'un même étoc, puisque la hauteur de coupe a un effet important sur l'effectif de rejets, et qu'il est classique que la croissance en diamètre soit relativement sensible à la concurrence (Assmann, 1970), y compris dans les taillis (Trimble, 1974; Lamson, 1983).

Les interactions constatées entre les effets bloc et traitement appellent plusieurs remarques quant à leur interprétation biologique. L'observation des tableaux II et IV met en évidence que les effets traitement moyens, qu'ils soient positifs ou négatifs, tendent à être principalement diminués dans le bloc 1 et renforcés dans le bloc 2. Le premier bloc étant le moins favorable à la croissance des rejets (tableaux II et IV), on peut interpréter l'interaction qui s'y manifeste par une moindre expression des effets des modalités de coupe en conditions de milieu limitantes. En ce qui concerne le bloc 2, on remarque (tableau I) que les modalités à plus forte interaction (hache bas et tronçonneuse) présentent le nombre de rejets par cépée le plus faible par rapport aux autres blocs. On peut donc penser à l'existence possible d'un effet limitant du nombre de rejets par cépée (compétition entre rejets) sur l'expression des effets des modalités de coupe, qui serait minimum dans le cas du bloc 2 .

Les résultats de la littérature concernant l'influence de la hauteur de coupe font apparaître un effet presque toujours nul de ce facteur sur la croissance en diamètre ou en hauteur ( $\mathrm{El}$ Houri Ahmed, 1977, sur eucalyptus; Debell et Alford, 1972, sur peuplier; Bélanger, 1979, sur platane; Cabanettes et Pagès, 1986, sur rejets dominants de châtaignier); les seuls effets constatés, qui vont dans le même sens que les nôtres, sont ceux obtenus par Crist et al (1983) sur peuplier et par Harrington (1984) sur aulne rouge, et ne sont observés que pour les 2 premières années de croissance. Par contre, l'effet de la hauteur de coupe sur le nombre de rejets est assez général (Khan et Tripathi, 1986; El Houri Ahmed, 1977; Bélanger, 1979, Crist et al, 1983; Harrington, 1984).

En ce qui concerne l'influence de l'outil de coupe, nous avons déjà observé l'influence de ce facteur pour le même dispositif sur la hauteur des maîtres rejets des cépées (Cabanettes et Pagès, 1986). Phillips (1971) observe une tendance peu significative sur la longueur de rejets de châtaignier, plutôt en faveur de l'outil hache; en revanche, il trouve une influence assez nette de l'outil sur le nombre de rejets, qui est au contraire plus élevé pour la modalité scie lors de la première année de croissance, la situation s'inversant ensuite dès la seconde année. Crist et al (1983), comparant l'effet de la scie et du sécateur sur de jeunes peupliers, ne notent aucun effet significatif sur la croissance ou le nombre de rejets.

Nous retiendrons de ces résultats leur complexité, intégrant la diversité biologique des espèces en cause (notamment en ce qui concerne le mode de répartition sur la base des tiges et la fragilité et la durée de vie des bourgeons dormants) et 
la diversité des modalités de coupe expérimentées dans chaque catégorie. Nos résultats obtenus sur jeunes rejets de châtaignier suggèrent qu'une coupe haute (30 $\mathrm{cm}$ au-dessus du sol) a pour conséquences la mise en jeu de plus nombreux bourgeons dormants donnant naissance à une population surnuméraire de petits rejets (dominance apicale partielle et/ou début de concurrence) dont la présence détermine une réduction des dimensions moyennes des rejets de la population totale. L'effet de l'outil de coupe, qui est toujours un effet positif de la hache par rapport à celui de la tronçonneuse, pourrait être lié à une hauteur de coupe pratiquée un peu plus basse qu'avec l'outil tronçonneuse; il pourrait aussi dépendre d'un moindre traumatisme (ou d'une stimulation?) de la souche (et des racines) avec la hache qu'avec la tronçonneuse (chauffage du bois, moindres vibrations).

En tout état de cause, il sera intéressant de suivre l'évolution de ces phénomènes aux stades ultérieurs de croissance car, comme le remarque très justement Johnson (1975), l'influence du milieu tend à prédominer avec l'âge du peuplement forestier.

\section{REMERCIEMENTS}

Que M. R. Tandy, qui a permis l'installation du dispositif sur sa propriété et qui nous a laissé toute liberté pour les interventions, soit ici vivement remercié.

\section{RÉFÉRENCES}

Assmann E (1970) The Principles of Forest Yield Study. Pergamon Press, Oxford

Astier R, Bouvier A, Coursol J, Denis JB, Dervin $C$, Jolivet $E$, Lesquoy $E$, Pons $O$, Tomassone $R$, Vila JP (1982) GENSTAT, un langage statistique. INRA, Station de biométrie, Paris
Bachacou J, Masson JP, Millier C (1981) Manuel de la programmathèque statistique AMANCE 81. INRA, Station de biométrie, Nancy

Bédéneau M (1988) Croissance du taillis de châtaignier en France: premiers résultats. Ann Sci For 45, 3 (sous presse)

Bélanger RP (1979) Stump management increases coppice yield of sycamore. South $J$ Appl For 3, 101-103

Blake TJ (1981) Dieback and stump senescence following decapitation of eucalypts in relation to auxin and phenols. Can J For Res 11, 2, 291-297

Blake TJ, Raitanen WE (1981) A summary of factors influencing coppicing. Int Energy Agency Rep NE 1981. 22

Cabanettes A (1986) Distribution des rejets de taillis par bouton et par étoc, comparaison de 4 espèces et évolution entre 1 et 5 ans. CR réunion du Groupe taillis, 20-21 mars 1986, Orsay, France. pp. 42-48

Cabanettes A, Pagès L (1986) Effet des techniques de coupe sur la hauteur des cépées dans un taillis de châtaignier (Castanea sativa Mill.) Can J For Res 16, 1278-1282

Crist JB, Mattson JA, Winsauer SA (1983) Effect of severing method and stump height on coppice growth. In: Intensive Plantation Culture: 12 Years Research. (Hansen EA, ed.), US Dep Agric For Serv Gen Tech Rep NC-91, 58-63

Dagnélie $P$ (1970) Théorie et méthodes statistiques. Applications agronomiques. 2, Duculot, Gembloux, Belgique

Debell DS, Alford LT (1972) Sprouting characteristics and cutting practices evaluated for cottonwood. Tree Plant Notes, 23, 1-3

El Houri Ahmed A (1977) The effects of stump heights on the coppicing power of Eucalyptus microtheca. Sudan Sylva 3, 22, 90-105

Harrington CA (1984) Factors influencing initial sprouting of red alder. Can $J$ For Res 14 , 357-361

Johnson PS (1975) Growth and structural development of red oak sprout clumps. For Sci 21, 413-418

Khan ML, Tripathi RS (1986) Tree generation in a disturbed sub-tropical wet hill forest of North-East India: effect of stump diameter and height on sprouting of four tree species. For Ecol Manage 17, 199-209

Lamson NI (1983) Precommercial thinning increases diameter growth of Appalachian hardwood stumps sprouts. J Appl. Forestry 93-97

Parade A (1860) Cours élémentaire de culture des bois. $4^{\mathrm{e}}$ ed., Grimblot-Raybois, Nancy 
Pagès $L$ (1986) Lois de croissance en biomasse du taillis: le robinier dans le Val-de-Loire. Ann. Sci. For. 43, 4, 533-550

Phillips JB (1971) Effect of cutting techniques on coppice regrowth. $Q J$ For $65,220-223$

Riedacker A, Knockaert C, Zaidi A (1985) Production ligneuse des taillis d'eucalyptus: effet de la fréquence de coupe et de la densité de plantation. Ann Sci For 42, $139-52$

Rinne P, Kauppi A, Ferm A (1987) Induction of adventitious buds and sprouts on beech seedlings (Betula pendula Roth and Betula pubescens Ehrh.). Can J For Res 17, 6, 545-555
Snedecor GW, Cochran WG (1957) Statistical Methods. 6th edition, The lowa State University Press, Ames, USA

Solomon DS, BLum BM (1967) Stump sprouting. US For Serv Res Paper NE-59

Taylor JS, Blake TJ, Pharis RP (1982) The role of plant hormones and carbohydrates in the survival of coppiced Eucalyptus seedlings. Physiol Plant 55, 421-430

Trimble GR (1974) Response to crop-tree release by 7-year-old stems of red maple stump sprouts and northern oak advance reproduction. US For Serv Res Paper NE-303 